\title{
LA INTEGRACIÓN ENERGÉTICA EN EL GRAN CHACO AMERICANO \\ DOI 10.29327/230731.12.24-6
}

Astrid Yanet Aguilera Cazalbón ${ }^{1}$

Resumen: En la región del Gran Chaco Americano confluyen áreas ricas en recursos energéticos correspondientes a Argentina, Bolivia, Brasil y Paraguay. La comercialización de energía, así como la infraestructura compartida, por ejemplo, en líneas de transmisión, gasoductos, o la construcción de usinas binacionales, fortalecen el proceso de integración regional entre los países que conforman la región. En el presente trabajo se busca analizar la situación actual y las perspectivas de integración de la infraestructura energética regional en el Gran Chaco, considerando algunos de los principales desafíos. Para ello, se utilizan como marco teórico de referencia algunos conceptos desarrollados por la Geopolítica Energética, desatacando la importancia del denominado Heartland suramericano, así como la centralidad de Bolivia dentro del proceso de integración energética y de la búsqueda de autonomía y de la autosuficiencia regional.

\section{Introducción}

El Gran Chaco Americano es una región de aproximadamente 1,14 millones de Km2 de América del Sur, distribuidos en el norte-central de Argentina, oeste de Paraguay, sureste de Bolivia y una pequeña parte de la región centro-oeste de Brasil. Se trata de un territorio con destacada relevancia en la geopolítica suramericana, por la riqueza de sus recursos y su potencial papel de integrador político y económico regional.

La disponibilidad de recursos naturales, cuya gestión y gobernanza debe ser compartida entre más de un país, es compleja, pero a su vez ofrece una posibilidad para que los países establezcan relaciones de cooperación mutua. La construcción de infraestructura en torno a esos recursos, como la infraestructura energética o la infraestructura de transportes, tiene carácter de largo plazo y tiende a ser una de las formas más eficientes de consolidar la integración física y evitar conflictos entre los países. Por ejemplo, actualmente existe gran interés por el desarrollo del corredor bioceánico que atraviesa la región del Gran Chaco. La infraestructura energética compartida (líneas de transmisión, gasoductos, entre otros) es fundamental para el aprovechamiento

\footnotetext{
${ }^{1}$ Licenciada en Economía Universidad Nacional de Salta (Argentina) - Alumna de maestría en Integración Contemporánea en América Latina - Universidade Federal da Integração Latino-Americana (Brasil) Becaria DS-UNILA

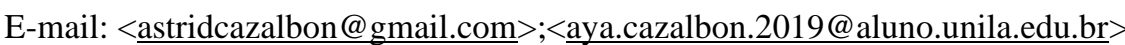


de los recursos energéticos disponibles, como gas natural y petróleo. De esa manera, estos recursos se tornan un instrumento clave de integración regional. Además, la comercialización de energía también puede promover la creación y ampliación de la infraestructura. La mayor disponibilidad de electricidad es beneficiosa no sólo porque posibilita y garantiza el consumo y la distribución de energía para uno residencial, sino también porque es clave para impulsar la matriz productiva local, cuyas actividades principales corresponden a la agricultura y ganadería.

El objetivo del presente trabajo es analizar la situación actual y las perspectivas de integración de la infraestructura energética de la región del Chaco Americano, considerando algunos de los principales desafíos para su consolidación. Para ello, se utilizan como marco teórico de referencia algunos conceptos desarrollados por la geopolítica energética. En la primera sección, se presentan los principales aspectos teóricos que señalan la importancia de la integración energética y de la centralización de las decisiones en relación al sistema energético, para conseguir la autosuficiencia y la seguridad energética regional. En la siguiente sección, se presenta un breve análisis de la geopolítica energética en el Heartland suramericano y sobre la centralidad de Bolivia dentro del proceso de integración energética. En la tercera sección, se analizan brevemente los antecedentes, situación actual y perspectivas de la integración de la infraestructura energética en la región del Chaco Americano. Por último, se dejan algunas consideraciones finales de todo lo abordado.

\section{HERRAMIENTAS TEÓRICO-CONCEPTUALES DE LA GEOPOLÍTICA ENERGÉTICA}

La geopolítica energética es un método de estudio que considera a los factores geográficos como importantes determinantes de la política gubernamental y de la posición relativa de poder de las naciones (Conant \& Gold 1981, p18). La comprensión del espacio geográfico con recursos energéticos importantes y el control de esos recursos, se tornan la base para una interpretación adecuada de esa posición (Kerr Oliveira, 2012). Sin embargo, al problematizar las disputas por los recursos energéticos se debe evitar caer en determinismos geográficos que sólo apunten a análisis parciales y fragmentados.

El campo de análisis de la geopolítica energética también estudia las disputas por recursos energéticos y la apropiación de tecnologías, el desarrollo 


\section{Universidade Federal Fluminense Instituto de Estudos Estratégicos}

de diferentes capacidades interestatales asociadas a los cambios tecnológicos, estrategias del poder corporativo, inclusive la militarización en torno a estos recursos. Otros elementos importantes, son la apropiación social de la energía y su papel en el modo de producción (Sauer, 2015), los conflictos ambientales y por mejoras en el acceso a la energía.

"A Geopolítica da Energia pode ser entendida como a análise do conjunto dos elementos geopolíticos e estratégicos que influenciam a exploração, a infraestrutura, o transporte e o uso final dos recursos energéticos" (Kerr Oliveira, 2012).

Hémery et al. (1993) y Kerr Oliveira (2012), trabajan con el concepto de Sistema Energético, procurando abarcar variables que vinculan la relación entre energía y sociedad, con características ecológicas, trayectorias tecnológicas, transiciones de poder en el Sistema Internacional y las diversas formas y condiciones de apropiación de los recursos energéticos. Es decir que, por ejemplo, aunque un mayor consumo de energía esté asociado a un crecimiento en los niveles de actividad, el sistema energético no puede reducirse meramente a la producción-consumo de ciertos volúmenes físicos de energía; éste reúne la compleja interrelación entre otras variables (TNI, 2019).

Diversos autores consideran la energía como un recurso de poder en el Sistema Internacional (Gomes Carmona, 2020; Kerr Oliveira, 2012). Sin embargo, la relación entre disponibilidad de recursos energéticos y riqueza no siempre es clara. Muchos países industrializados, que son actores claves en las disputas por recursos energéticos, se apropian de los recursos de países menos industrializados por intermedio de diversas formas de poder, como el poder de los medios de pago, el control de la tecnología, del transporte y de la infraestructura (Conant \& Gold 1981; Kerr Oliveira 2012; Fuser 2013).

En la mayoría de los lugares en donde se encuentran disponibles los recursos (principalmente hidrocarburos) se dan puntos de estrangulamiento, que son susceptibles de interrupciones, bloqueos y conflictos armados. Por ello, la seguridad y la defensa, tanto en las regiones productoras como en las regiones consumidoras son de importancia estratégica (Conant \& Gold 1981, p64). En ese sentido, la infraestructura de energía es considerada un tipo de infraestructura crítica; toda estrategia al respecto debe contemplar un nivel de análisis geopolítico y de la distribución geográfica de los recursos (Aguilera Cazalbón, 2020).

La disponibilidad de fuentes de energía en un determinado lugar no es una condición que asegure el abastecimiento local. Existen innúmeros ejemplos de usinas localizadas en regiones periféricas que disponen de recursos energéticos para la generación, pero no cuentan con infraestructura adecuada 
para la distribución y el consumo local. El destino de la electricidad generada en esas áreas acaba en las grandes metrópolis y los polos industriales, que concentran un gran número de habitantes y de industrias de sectores productivos intensivos en el uso de electricidad. También existen zonas rurales en las que el acceso a la energía es limitado o está fuera del sistema de interconectado de la red eléctrica.

A diferencia de otros bienes, la electricidad es un bien de consumo inmediato. Solo se produce lo que se va a consumir porque no se puede almacenar en grandes cantidades ${ }^{2}$. Por ello, toda la infraestructura, que abarca también las redes de transmisión y distribución, son esenciales para garantizar la universalización del acceso a la electricidad, tornándose una variable clave en el desarrollo económico de un país o región (Kerr Oliveira, 2012).

Las políticas de infraestructura también deben considerar la existencia de regiones periféricas y de asimetrías entre las diversas regiones. Cuando se dispone de una infraestructura de calidad y que considera heterogeneidades espaciales, estas pueden modificar la distribución espacial de las actividades productivas en una determinada región (Padula, 2012). La incorporación de las regiones periféricas a los circuitos productivos, sugiere mayor atención al planeamiento de la infraestructura integrada. De acuerdo con Costa (2011), siempre hay una interconexión entre el sentido geográfico y político, geopolítico y geoeconómico de la integración con la organización y el aprovechamiento de los espacios.

\section{Estrategia Energética}

La Estrategia Energética se refiere a la estrategia que un Estado o conjunto de Estados puede elaborar para enfrentar los problemas en el sector energético. De acuerdo con Kerr Oliveira (2012) la Estrategia Energética puede clasificarse principalmente en tres categorías, a saber: (1) la estrategia de autonomía o autosuficiencia energética, que consiste básicamente en la diversificación de la matriz energética, la descentralización de la infraestructura de generación y distribución de energía, la innovación energética y la eficiencia energética; (2) la estrategia del aumento de la seguridad en el abastecimiento externo de energía, que se alcanza a través de la diversificación de los proveedores externos y la militarización del control de recursos energéticos en el exterior; y (3) la estrategia de la integración energética regional, que se

\footnotetext{
${ }^{2}$ https://fundacionsolon.org/2020/01/23/electricidad-bien-de-consumo-inmediato/
} 


\section{Universidade Federal Fluminense Instituto de Estudos Estratégicos}

puede alcanzar mediante diversos mecanismos de cooperación, creación y fortalecimiento de instituciones promotoras de la integración energética.

\section{Estrategia de Integración de la Infraestructura Energética}

La creación y ampliación de infraestructura tiene impacto sobre el desarrollo económico al expandir la capacidad productiva y crear condiciones propicias para que todos los sectores de la economía puedan operar. Estos impactos, además de contar con economías de escalas, serían potencializados si se considera a una región como un todo y se busca la integración de la infraestructura regional. Para ello, se debe aprovechar al máximo las potencialidades regionales en favor de la autonomía, conectando los espacios económicos de producción y consumo, posibilitando la inserción de regiones más atrasadas al desarrollo, interconectándolas al mercado (Costa, 2011).

Padula (2010) sostiene que la integración energética regional provee seguridad y autonomía energética, aumentando su poder en el sistema internacional. Asimismo, promueve la industrialización y el desarrollo en los países que conforman la región, impactando en la generación de empleo y renta, a través de una mayor disponibilidad de energía, especialmente en las industrias intensivas en energía. Por otro lado, permite resolver los problemas de déficit de energía de algunos países a través de los intercambios comerciales de energía. En ese sentido, el autor defiende la idea de que la integración de la infraestructura y la ocupación de espacios, tienen un papel central en el proceso de integración regional, y deben ser encarados desde una concepción políticoestratégica, que no se restrinja meramente a una visión económica, comercial dominada por las fuerzas de mercado (Padula, 2012).

En la región sudamericana, compuesta por países con gran heterogeneidad en las diferentes dimensiones del desarrollo, la importancia de la integración de la infraestructura de la energía cobra mayor relevancia dada la posibilidad de abaratar costes entre los diversos países, aprovechar complementariedades y coordinar acciones en conjunto. Algunos autores destacan también la importancia que esta tendría para alcanzar la autonomía en las decisiones energéticas de la región. Además, las diversas fuentes disponibles están distribuidas de tal forma que potencializan los beneficios posibles de su aprovechamiento a través de la integración energética regional, en función de la complementariedad de las fuentes entre los diferentes países.

Según Kerr Oliveira (2012), la integración regional es una solución estratégica para los bloques de países como los de América del Sur, que buscan más autonomía y soberanía en el Sistema Internacional, y necesitan ampliar la 
generación de empleo, de renta y la capacidad de consumo promedio de la población. En ese sentido, según el autor, la integración regional podría garantizar un mayor grado de desarrollo económico, tecnológico e industrial con la ampliación de la infraestructura.

La infraestructura integrada podría impulsar las cadenas productivas locales y tornarlas más competitivas, por la disminución de los costos logísticos. El consumo interno sería beneficiado con la hipotética reducción de precios. No obstante, como fue señalado anteriormente, la región sudamericana está caracterizada por grandes heterogeneidades estructurales. Existen dentro de ella muchas sub regiones que no están incorporadas a los circuitos productivos por estar alejadas de los puertos o de los centros urbanos e industrializados. Para Paz (2011) los espacios y redes de integración en América del Sur, cargan una herencia de una economía históricamente agroexportadora. De ese modo, la infraestructura se encuentra concentrada en torno a los puertos de exportación, con muy bajo nivel de integración entre las economías nacionales. Considerando este último aspecto, para Padula (2010):

"Na América do Sul, como espaço continental, a ação planejada e integrada em infra-estrutura tem que obrigatoriamente buscar três ações (Os "3 Is"), conforme exposto em Costa \& Padula (2007): Induzir o desenvolvimento - em espaços isolados e/ou menos desenvolvidos; Integrar os mercados já desenvolvidos, criando sinergias; Irrigar economicamente os espaços desenvolvidos (metrópoles e centros econômicos) que se encontram saturados (sofrem com deseconomias de aglomeração e congestionamentos)" (PADULA, 2010).

Además, la integración energética es fundamental para establecer un vínculo cooperativo entre los países. Sin embargo, es necesario indagar en qué medida los proyectos de infraestructura integrada buscan la autosuficiencia, la seguridad y el desarrollo para la región, o esconden por detrás las demandas impuestas por agentes con intereses concretos involucrados en la creación de determinadas obras de infraestructura. Esto refuerza la necesidad de planeamiento y de la elaboración de una estrategia conjunta.

Uno de los limitantes para impulsar el proceso de integración de infraestructura, está relacionado a las diferentes asimetrías que caracterizan a los Estados de la región en diversos aspectos: población, nivel de producto, recursos naturales, niveles de ingreso, tipo de gobierno, y su relevancia en el orden internacional. Asimismo, esto condiciona las acciones coordinadas de planeamiento y ejecución de proyectos de infraestructura. Aunque existieron diversos intentos de planeamiento conjunto y de centralización de las decisiones sobre infraestructura como la Iniciativa para la Integración de la Infraestructura Suramericana (IIRSA) y el Consejo Suramericano de Infraestructura y 


\section{Universidade Federal Fluminense Instituto de Estudos Estratégicos}

Planeamiento (COSIPLAN), como señala Padula (2010), es difícil discutir un modelo de infraestructura para la región de forma separada a una concepción de desarrollo regional, nacional y espacial. Por otra parte, estas siempre se han enfrentado las crisis políticas y coyunturales que han obstaculizado sus avances.

\section{Centro de Decisión Energético}

El concepto de Centro de Decisión Energético es fundamental para la consolidación de la estrategia energética:

"O Centro de Decisão Energético como a capacidade de planejar, operar, modificar e implementar uma Estratégia para o desenvolvimento completo $e$ integrado de diferentes setores da economia e da sociedade que dependem ou estão relacionados diretamente à Energia. Seria a capacidade de planejar e materializar uma Política Energética, ou uma Estratégia Energética, necessária para o desenvolvimento de todas as demais atividades produtivas e logísticas de um país ou bloco de países. O Centro de Decisão Energético é o que permite viabilizar uma Estratégia de Segurança Energética de longo prazo, com continuidade no tempo e no espaço, integrando de forma eficiente os diversos sistemas energéticos, de transportes e comunicações com os sistemas produtivos de um país ou região" (Kerr Oliveira, 2012).

Según esta perspectiva, la consolidación de un Centro de Decisión Energética en América del Sur es fundamental como estrategia de desarrollo, que sea capaz de concentrar las decisiones energéticas con autonomía regional. Este concepto, que contempla a la energía de manera sistémica, considera la matriz energética de producción y consumo, la infraestructura, el modelo productivo, la capacidad de integración de cadenas de valor local, entre otras variables centrales. La integración energética configura entonces una alternativa que puede contribuir para asegurar la soberanía energética la región (Kerr Oliveira, 2012; Padula, 2010).

Aunque existe gran relevancia geopolítica de la cuestión energética para los países de América del Sur, la integración energética como un sistema de interconexión estratégica de las redes de producción y consumo de energía entre los países suramericanos, es un proceso todavía muy incipiente en América del Sur (Paz \& Nunes, 2011). 


\section{LA GEOPOLÍTICA ENERGÉTICA EN EL HEARTLAND SUDAMERICANO}

\section{El concepto de Heartland en América del Sur}

El término Heratland, que significa "corazón de la tierra" o "corazón del mundo", fue empleado por primera por el geógrafo inglés Halford Mackinder en 1919. Este concepto se utilizó en la reformulación de su artículo "The Geographical Pivot of History", en donde hacía referencia a un área pivot y presentaba su teoría geopolítica y estratégica del poder terrestre (Mello, 1999). Según su interpretación, el mundo estaría dividido en tres zonas: el Gran Océano (que abarca tres cuartos del planeta), la Isla Mundial (Europa, Asia y África) y las islas-continentes menores (Australia y América) (Mckinder, 2010)․

"A grande área continental, cujo centro estava afastado dos mares, tinha características geográficas de uma fortaleza natural rodeada por grandes acidentes geográficos, como rios, deserto, geleiras, planaltos e cadeias montanhosas. Além disso, essa região oferecia a possibilidade de fácil projeção em diversas direções. Tinha poder de articulação territorial e estava posicionada no centro de uma grande massa continental (PFRIMER; ROSEIRA, 2009, p.5) " (Wexell Severo, 2012)

Según Kerr Oliveira \& Leite García (2010), el Heartland también se caracteriza por ser una región sub-poblada:

"O Heartland foi definido originalmente como um e vasto território, com amplo potencial para a agricultura, pecuária, extrativismo ou assentamento de grupos humanos. Rica em recursos naturais de toda sorte, além de terras férteis ou potencialmente agricultáveis, vastas reservas de recurso hídricos, planícies, estepes e florestas, a zona "pivô" da Eurásia incluía, em suas definições originais, a idéia de rios caudalosos, enormes jazidas de recursos minerais metálicos e não metálicos, do ferro e manganês ao fósforo e potássio, mas também muito relevante, enormes reservas de recursos energéticos como carvão mineral e petróleo, este último principalmente na região do Mar Cáspio. Neste sentido, a disponibilidade de recursos energéticos parece, à luz da atualidade, central naquele conceito original" (Kerr Oliveira \& Leite García, 2010).

Durante el siglo pasado, el concepto de Heartland fue revisitado por una serie de autores de América del Sur que destacaron su potencial en recursos naturales, como recursos hídricos, gas natural, petróleo, minerales y tierras fértiles. Entre las principales contribuciones se encuentran los trabajos de Travassos (1935), Tambs (1965) y posteriormente los trabajos de Couto e Silva

\footnotetext{
${ }^{3}$ Traducción realizada de la Conferencia pronunciada ante la Real Sociedad Geográfica (Londres), el 25 de enero de 1904; reproducida posteriormente en The Geographical Journal, vol. 23, nº. 4, pp.421-437.
} 


\section{Universidade Federal Fluminense Instituto de Estudos Estratégicos}

(1955) y Pinochet (1968) (Wxell Severo, 2012). Estos autores desarrollaron diversas herramientas de análisis teóricas, en algunos casos, asociándolas directa o indirectamente al concepto de Mackinder. Algunos ejemplos son el concepto de "área de soldadura", desarrollado por Couto e Silva, o el concepto de Triángulo Estratégico de Travassos (1935) (Kerr Oliveira \& Leite García, 2010; Wexell Severo, 2012).

Según Kerr Oliveira \& Leite García (2010), asociar el concepto de Heartland de Eurasia a la región suramericana es una herramienta útil para el análisis de las políticas y estrategias de seguridad, defensa e integración, sea de manera unilateral, por parte de cada país, o en términos regionales. Los autores sostienen también que este concepto destaca la centralidad de los territorios de Bolivia y Paraguay, además del norte de Argentina y centro-oeste de Brasil, para pensar en una región absolutamente vital para el proceso de Integración suramericana. Por otra parte, señalan que contribuye a repensar una estrategia basada en la centralidad de los proyectos de construcción de infraestructura física común en los países suramericanos (transporte, energía y comunicaciones) especialmente a través del área Pivó o corazón del continente. Sin embargo, Wexell Severo (2012) advierte que este concepto aplicado a Suramérica tiene algunas limitaciones, principalmente en relación a la ausencia de un poder integrador político, económico y bélico.

La figura 1, muestra el mapa de Suramérica dividido en macro-regiones. El Norte Amazónico, el Escudo Andino, la región Noreste, el Cono Sur, la Península Austral y en el centro se ubica el Heartland suramericano.

\section{La importancia estratégica de Bolivia y la Geopolítica energética en el Heartland suramericano}

Según Wexell Severo (2012), Mackinder usó la idea de Heartland para referirse a un área mediterránea central que daría al Estado que la domina capacidad para movilizarse y para proyectarse en todas las direcciones da Eurasia. Al mismo tiempo este contaba con una fortaleza natural.

"O caso da Bolívia é similar. Uma área mediterrânea central que pode ser considerada uma fortaleza natural; um altiplano entre duas cordilheiras. É uma plataforma de projeção para todas as direções e, ao mesmo tempo, está sujeita a sofrer ameaças desde todas as direções. A grande boliviana questão é a não utilização destas potencialidades pelo Estado" (Wexell Severo, 2012).

De acuerdo a la visión de Mario Travassos (1935) Bolivia sería el único país de América del Sur en ocupar simultáneamente o ejercer proyección sobre cuatro espacios relacionados a su localización (Cordillera de los Andes, que 
divide al continente de este a oeste, y las cuencas de los ríos Amazonas y del Plata, que condicionan una división norte-sur) (Wexell Severo, 2012).

Figura 1: El Heartland de América del Sur y las macro-regiones del continente

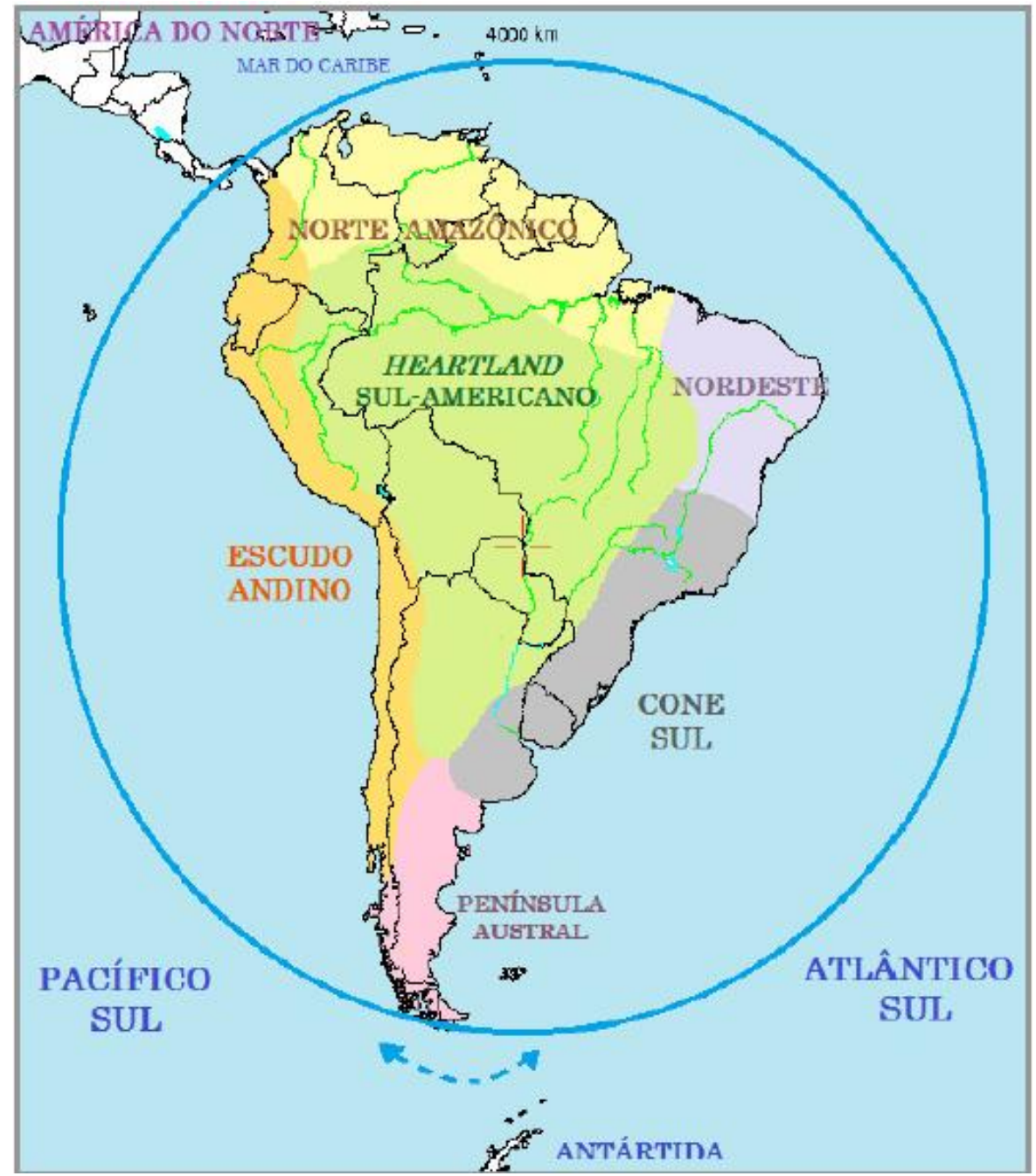

Fuente: Kerr Oliveira \& Leite García (2010)

Si bien la porción que ocupa Bolivia en la región del Chaco Suramericano es reducida respecto de los otros países, es de suma importancia considerar la teoría del Heartland suramericano y la centralidad de Bolivia dentro del proceso de integración regional ${ }^{4}$. Además de su localización geográfica, que como fue mencionado anteriormente, le permiten a Bolivia el acceso a diversos espacios y la posibilidad de proyectarse en varias direcciones, la abundancia de recursos energéticos es uno de los principales motivos por los que Bolivia es uno de los

\footnotetext{
${ }^{4}$ Por ejemplo, Wexell Severo (2012) destaca la relevancia de la ciudad de Santa Cruz de la Sierra como un actual centro de articulación continental e importante motor económico de Bolivia, que además presenta una intensa integración con el estado brasileño de Mato Grosso en diversos sectores, como el agrícola y el energético. Se trata de una macro región que tiene salida hacia los océanos Pacífico y Atlántico.
} 


\section{Universidade Federal Fluminense Instituto de Estudos Estratégicos}

países más importantes para el proceso de integración regional. Con frecuencia, en la literatura revisada los conflictos en territorio boliviano están asociados a la extracción y uso de recursos energéticos. Por ejemplo, las tensiones en el Triángulo estratégico al que se refería Travassos, situado entre las ciudades de Santa Cruz, Cochabamba y Sucre, en donde existían ciudades con minas de Oruro y Potosí (Kerr Oliveira \& García Leite, 2010). Existen también otros indicios de conflictos por recursos energéticos en el área:

"Alguns autores, como Chiavenato (1980), relacionam o conflito com as descobertas de jazidas de petróleo no Chaco, por volta dos anos 1920. Como na Bolívia as explorações eram feitas pela estadunidense Standard Oil, enquanto no Paraguai os trabalhos estavam a cargo da inglesa Shell, a guerra seria resultado dos interesses dessas petrolíferas" (Wexell Severo, 2012).

Los recursos energéticos del Heartland sudamericano (recursos hídricos, gas natural, petróleo) pueden ser considerados estratégicos por su riqueza y ubicación geográfica (Wexell Severo, 2012). A los fines de este trabajo, es importante la observación que realizan Kerr Oliveira \& Leite García (2010), acerca de la centralidad de la cuestión energética en el concepto de Heartland. No obstante, los autores también identificaron que esa perspectiva fue bastante desconsiderada en los análisis, por ejemplo, en el concepto de área de Soldadura de América del Sur (que comprende al área del Heartland) desarrollado por Couto e Silva, no se consideran como tan relevantes los recursos energéticos potenciales existentes en esa región, incluyendo el potencial hidroeléctrico e indirectamente el potencial hidroviario, las ricas reservas de gas natural y la mayor reserva de litio conocida en el mundo, que se encuentra en Bolivia.

El aprovechamiento y el uso eficiente del sistema energético-productivo permite mayores posibilidades de desarrollo económico, por ende, las estrategias de centralización de las decisiones a través del planeamiento para el uso compartido de la infraestructura energética constituyen una de las formas más destacadas para alcanzar un mayor aprovechamiento de los recursos. En la región, la Iniciativa para la Integración de la Infraestructura Suramericana (IIRSA), que posteriormente pasaron a la administración del COSIPLAN, se tornaron las políticas más importantes en pos de articular la integración física, ampliando las posibilidades de comercio intrarregional. Inclusive, porque viabiliza el acceso tanto al Pacífico como al Atlántico.

Según Wexell Severo (2012), en ese contexto, la importancia del territorio boliviano solo tiende a aumentar, ya sea desde la perspectiva propuesta por Travassos pero con un nuevo triángulo estratégico conformado por ciudades 
que en la actualidad tienen más preponderancia (Santa Cruz, Cochabamba e Tarija) que las analizadas originalmente, o, junto a Paraguay y al Centro-Oeste de Brasil, Bolivia continuará teniendo una función crucial en este proceso, como la gran "área de soldadura".

\section{INFRAESTRUCTURA ENERGÉTICA EN EL CHACO AMERICANO}

\section{La relación entre la matriz productiva y la energía}

América del Sur es una región favorablemente dotada de recursos energéticos, tanto en términos de combustibles fósiles (petróleo, gas, carbón) y renovables (hidroeléctrica, eólica, solar, biomasa y geotérmica). La región tiene aproximadamente el $19 \%$ de las reservas mundiales de petróleo, el $4 \%$ de las reservas de gas natural y casi el 1,6\% de las reservas de carbón (OIES, 2016).

La región chaqueña, como unidad ambiental, tiene límites naturales que no coinciden con los límites políticos establecidos por las naciones. Los componentes naturales de esta región son compartidos más allá de las fronteras políticas (Investigación para el Desarrollo, 2017). La coordinación entre los países que conforman el área es fundamental para el aprovechamiento de los recursos energéticos que dispone. La comercialización de energía, así como la infraestructura compartida, por ejemplo, en gasoductos, líneas de transmisión o la construcción de usinas binacionales, además de potencializar la integración física, fortalecen el proceso de integración regional en los países que conforman la región.

Comprender el sistema energético en su conjunto, implica también comprender el modelo productivo asociado. Las principales actividades económicas desarrolladas en la región corresponden a la agricultura y ganadería, con excepción de Bolivia, donde la preeminencia se observa en la producción de hidrocarburos y en segundo lugar se ubican las actividades agropecuarias. La región es considerada clave en la producción de alimentos a nivel global y altamente competitiva debido a la incorporación de tecnologías de producción y gestión. Por ejemplo, Argentina se destaca en la producción de soja, girasol, maíz y trigo; Paraguay se destaca en la producción de carne, maní y maíz; y Bolivia es también considerado como un importante productor de cereales. El cultivo de soja se expandió sustancialmente en los últimos años, principalmente por su elevada rentabilidad, avanzando sobre las tierras forestales de la región chaqueña. La mayor parte de la soja producida es de tipo transgénica y se exporta principalmente a Europa y Asia (Investigación para el Desarrollo, 2017). 


\section{Universidade Federal Fluminense Instituto de Estudos Estratégicos}

La energía generada en el gran Chaco es distribuida hacia diversas áreas. La atención de las demandas de importantes regiones industriales brasileñas (como la región sur y parte del Estado de São Paulo) es realizada en gran parte por el gas natural boliviano transportado a través del GASBOL, lo que hace a esta fuente de oferta ser relevante para Brasil. Además de la importancia para la atención del mercado consumidor brasileño, la venta de gas natural es significativa para Bolivia, pues representa más del $50 \%$ de sus exportaciones (EPE, 2017).

La población está caracterizada por una distribución muy heterogénea. Viven aproximadamente ocho millones novecientas mil personas (8,9 millones). En las zonas periféricas del Gran Chaco se encuentran las mayores concentraciones de población, en donde la densidad llega a más de 40 habitantes por kilómetro cuadrado, territorios correspondientes a centros urbanos de Bolivia y Argentina. En cuanto a la distribución de población rural, está íntimamente relacionada a los modos y formas de producción, fundamentalmente la agropecuaria (Investigación para el Desarrollo, 2017).

Intereses de diversos actores públicos y privados confluyen en torno a la infraestructura y los recursos que dispone el chaco. Un claro ejemplo en la actualidad es la construcción del corredor bioceánico que atraviesa la región, por el cual se transportarían, principalmente, mercaderías y diversos bienes que se producen en la región. Esto a su vez podría demandar más energía, no sólo para el abastecimiento del trasporte que circule por ese corredor, sino también porque podría dinamizar las economías regionales y las zonas productoras. Por otra parte, es importante considerar el potencial de los ejes de Integración y Desarrollo del COSIPLAN. El Eje Capricornio atraviesa gran parte de la región del chaco. Se extiende por la costa del Pacífico de Chile, la región andina de Bolivia, el norte de Argentina, todo Paraguay y los Estados de Brasil en la costa atlántica (Rio Grande do Sul, Santa Catarina, Paraná y una porción de Mato Grosso do Sul). Las obras de este eje están orientadas a fortalecer la conectividad de los territorios involucrados hacia el Pacífico y hacia la Hidrovía Paraguay-Paraná y a mejorar las condiciones de integración productiva y competitivas del Noroeste de Argentina, Sur de Bolivia y Paraguay 5 .

\footnotetext{
${ }^{5}$ http://www.iirsa.org/
} 


\section{Infraestructura energética en el Gran Chaco: Antecedentes, situación actual y perspectivas}

\section{Infraestructura gasífera}

Morales Udaeta (et al., 2006), señalan que el gas natural es un recurso estratégico que configura uno de los principales instrumentos de Integración regional en el Cono Sur. Este brinda la posibilidad de una mayor interconexión energética física debido a que su papel fue evolucionando en función a sus ventajas en comparación a los otros combustibles fósiles. Estos autores también destacan que se debe considerar el potencial de las reservas disponibles y sus posibles necesidades de complemento transfronterizo de varios países productores, así como la integración de los mercados eléctricos y gasífero.

Los primeros indicios de integración gasífera en la región se dieron justamente entre Argentina y Bolivia, dentro del área del Gran Chaco, en la década de 1970 con el gasoducto Yabog. Hasta mediados de 1990, era el único gasoducto transfronterizo en la región. En 1999, comenzó a funcionar el gasoducto Gasbol que conecta Bolivia con Brasil (OIES, 2016), a través del cual Bolivia exporta gas natural a Brasil, garantizando el abastecimiento de distribuidoras de gas, termoeléctricas y refinerías. Brasil pasó a importar volúmenes crecientes del gas de Bolivia, no solo por el GASBOL, sino también por el gasoducto Lateral Cuiabá, que inició sus operaciones en 2001 (EPE, 2017). El Gasbol Inicia en la ciudad boliviana de Santa Cruz de la Sierra y recorre 136 municipios en 6 Estados brasileños (Mato Grosso do Sul, São Paulo, Paraná, Santa Catarina y Rio Grande do Sul) hasta llegar a Canoas. Se trata del mayor gasoducto de América Latina en extensión, con un total de 3.150 kilómetros, siendo $557 \mathrm{~km}$ en Bolivia y $2.593 \mathrm{~km}$ en Brasil ${ }^{6}$ (CBIE, 2020).

Hacia el año 2000, Argentina comenzó a exportar gas a Brasil, tratándose de un proyecto que pretendía suministrar gas al sur de Brasil y a su vez competir con el gas boliviano que comenzó a fluir hacia Brasil por esa misma época a través del gasoducto Gasbol (OIES, 2016). A inicios del año 2010, el gas que se transportaba de Bolivia a Brasil era responsable por cerca de 1/3 de la oferta total de Brasil, pero la instabilidad en el suministro redujo la participación para un poco más del 25\% entre 2016 y 2018 (CBIE, 2020). Caber destacar que Bolivia tuvo un aumento de siete veces en las reservas probadas de gas en solo

\footnotetext{
${ }^{6}$ El trecho norte tiene una extensión de 1.147 km y conecta Corumbá con Guararema, La conexión entre São Paulo y Rio Grande do Sul se da en el trecho sur. Entre las ciudades de Paulínia y Canoas, el gasoducto recorre $1.176 \mathrm{~km}$ (https://petrobras.com.br/pt/nossas-atividades/principais-operacoes/gasodutos/)
} 


\section{Universidade Federal Fluminense Instituto de Estudos Estratégicos}

trece años (1998-2011). Mientras que, en Brasil, más del 80\% de las reservas están ubicadas frente a la costa.

En general, los intercambios transfronterizos se correspondieron más con iniciativas de interconexiones bilaterales entre productores y consumidores que con una estructura de mercado verdaderamente regional, armonizado en cuestiones regulatorias, precios y políticas. Los gasoductos existentes han creado la base para una red de transporte de gas subregional, pero no han logrado consolidar una integración regional más amplia (OIES, 2016). Según Morales Udaeta (et al., 2006), la integración energética fue implementada más por fuerza de las circunstancias que por la decisión conjunta de un proceso pensado para el desarrollo harmónico de la región. Hubo una serie de complicaciones tales como reducciones en volúmenes, dificultades en las negociaciones de precios, tiempo incierto para desarrollo de nuevas reservas en países exportadores y relaciones políticas cambiantes. Por otro lado, en épocas de escasez, los países productores dieron prioridad a sus mercados internos. Esto llevó a los países importadores a buscar nuevas fuentes de importación de gas y recurrieron al Gas Natural Licuado (GNL) para aumentar la seguridad del suministro, agregar volúmenes adicionales y brindar mayor flexibilidad (OIES, 2016).

En el Cono Sur, que comprende parte del Gran Chaco, hubo algunos problemas en la comercialización de gas entre los países. Uno de los más significativos, fue la disminución de producción de gas en Argentina en el año 2004, que acabó afectando a Chile, Brasil y Uruguay por la interrupción en el suministro y suspensión de contratos, para darle prioridad al abastecimiento interno. Posteriormente, Argentina reinició las importaciones desde Bolivia y firmó un nuevo contrato de 20 años en $2006^{7}$. En relación a las exportaciones de gas de Bolivia a Brasil, si bien la provisión de gas natural por Bolivia ha sido constante, la estrategia brasilera de reducción de la dependencia del gas natural boliviano (a través de la implantación de terminales de regasificación de GNL, ampliación de la infraestructura de transporte de gas natural y desarrollo de la producción de campos de gas natural) constituye un desafío para Bolivia por el aumento de la competencia en el abastecimiento de este commodity, vía otras alternativas de oferta para Brasil, como el GNL importado y el gas natural oriundo del Pré-Sal (EPE, 2017).

\footnotetext{
${ }^{7}$ El nuevo contrato exigía un aumento constante de los envíos de gas natural y oleoducto adicional entre los dos países (el Gasoducto Integración Juana Azurduy (GIJA) que entró en línea en 2011. El norte de Argentina no está bien abastecido por la red nacional de transporte de gas natural y depende de productos petroleros más costosos (el gas boliviano está destinado a reemplazar diésel y gas licuado de petróleo). El Gasoducto del Noreste Argentino (GNEA) es otro oleoducto transfronterizo en construcción que llega a la región noreste y recibirá Gas boliviano proveniente del Gasoducto Juana Azurduy (http://www.ieasa.com.ar/index.php/proyectos/gnea/).
} 
Tabla 1: Gasoductos Transfronterizos dentro del área del Gran Chaco, 2016

\begin{tabular}{|c|c|c|c|c|c|c|}
\hline Nombre & Origen & Destino & $\begin{array}{l}\text { Año de } \\
\text { ejecución }\end{array}$ & Tamaño & Capacidad & $\begin{array}{l}\text { Situación } \\
\text { a } 2015\end{array}$ \\
\hline $\begin{array}{l}\text { Yabog } \\
\text { (Yacimientos } \\
\text { Bolivian Gulf) }\end{array}$ & $\begin{array}{l}\text { Bolivia } \\
\text { (Santa } \\
\text { Cruz de la } \\
\text { Sierra) }\end{array}$ & $\begin{array}{l}\text { Argentina (Red } \\
\text { de Transporte } \\
\text { Norte, Campo } \\
\text { Durán) }\end{array}$ & 1972 & $441 \mathrm{~km}$ & $\begin{array}{l}6 \text { bcma } \\
\text { (aumentad } \\
\text { o a } 10 \\
\text { bcma em } \\
2015 \text { ) }\end{array}$ & Utilizado \\
\hline $\begin{array}{l}\text { Gasoducto } \\
\text { Integración } \\
\text { Juana } \\
\text { Azurduy } \\
\text { (GIJA) }\end{array}$ & Bolivia & $\begin{array}{l}\text { Argentina } \\
\text { (Salta } \\
\text { Province) }\end{array}$ & 2011 & $50 \mathrm{~km}$ & $\begin{array}{l}7.7 \mathrm{mcm} / \mathrm{d} \\
(2.7 \mathrm{bcma})\end{array}$ & Utilizado \\
\hline $\begin{array}{l}\text { Gasoducto } \\
\text { Noreste } \\
\text { Argentina }\end{array}$ & $\begin{array}{l}\text { Gasoducto } \\
\text { de Bolivia } \\
\text { GIJA }\end{array}$ & $\begin{array}{l}\text { Argentina } \\
\text { (Provincias del } \\
\text { Centro y del } \\
\text { Norte: Salta, } \\
\text { Formosa, } \\
\text { Chaco, } \\
\text { Misiones, Entre } \\
\text { Ríos y Santa } \\
\text { Fé) }\end{array}$ & 2016 & $1810 \mathrm{~km}$ & $\begin{array}{l}27.7 \\
\mathrm{mcm} / \mathrm{d} \\
(10.1 \\
\mathrm{bcma})\end{array}$ & $\begin{array}{l}\text { En } \\
\text { construcci } \\
\text { ón }\end{array}$ \\
\hline Gasbol & $\begin{array}{l}\text { Bolivia } \\
\text { (Santa } \\
\text { Cruz de la } \\
\text { Sierra) }\end{array}$ & $\begin{array}{l}\text { Brasil (Sur, } \\
\text { Porto Alegre en } \\
\text { Rio Grande do } \\
\text { Sul) }\end{array}$ & $1999-2000$ & $3150 \mathrm{~km}$ & $\begin{array}{l}30 \mathrm{mcm} / \mathrm{d} \\
(10.9 \\
\text { bcma })\end{array}$ & $\begin{array}{l}\text { Terminad } \\
\text { o }\end{array}$ \\
\hline Bolivia-Brasil & $\begin{array}{l}\text { Bolivia } \\
\text { (Rio San } \\
\text { Miguel) }\end{array}$ & $\begin{array}{l}\text { Brasil (Cuiabá } \\
\text { en el Estado de } \\
\text { Mato Grosso) }\end{array}$ & 2002 & $626 \mathrm{~km}$ & $\begin{array}{l}2.8 \mathrm{mcm} / \mathrm{d} \\
(1 \mathrm{bcma})\end{array}$ & $\begin{array}{l}\text { Terminad } \\
0\end{array}$ \\
\hline
\end{tabular}

Fuente: Oxford Institute Energy Studies (2016).

Para Morales Udaeta (et al., 2006), la demanda reprimida de gas natural en el área del Gran Chaco demostraba las necesidades diarias significativas y la consecuente necesidad de incrementar la oferta. El problema de la demanda reprimida aún persiste hasta los días actuales. Según EPE (2017), existe cierta desconfianza por parte de Brasil en relación al gas de Bolivia, por la posibilidad de que este último no pueda hacer frente a sus compromisos de proveer el gas que Brasil demanda.

Tanto las interrupciones, como los problemas de seguridad en el abastecimiento y los desafíos que representa la búsqueda de alternativas de los países consumidores de gas natural para los países productores, se podrían evitar con mecanismos de cooperación eficientes. A pesar de todo, dentro del Cono Sur hay relativamente buena interconectividad, con una decena de gasoductos transfronterizos entre Bolivia, Argentina, Chile y Uruguay. En la Tabla 1 se muestran los gasoductos transfronterizos dentro del área del Gran Chaco existentes hasta 2016, y en la figura 2 se muestra situación de la infraestructura gasífera en América del Sur hacia el año 2016. 
Figura 2: Infraestructura de Gas Natural en Suramérica, 2016

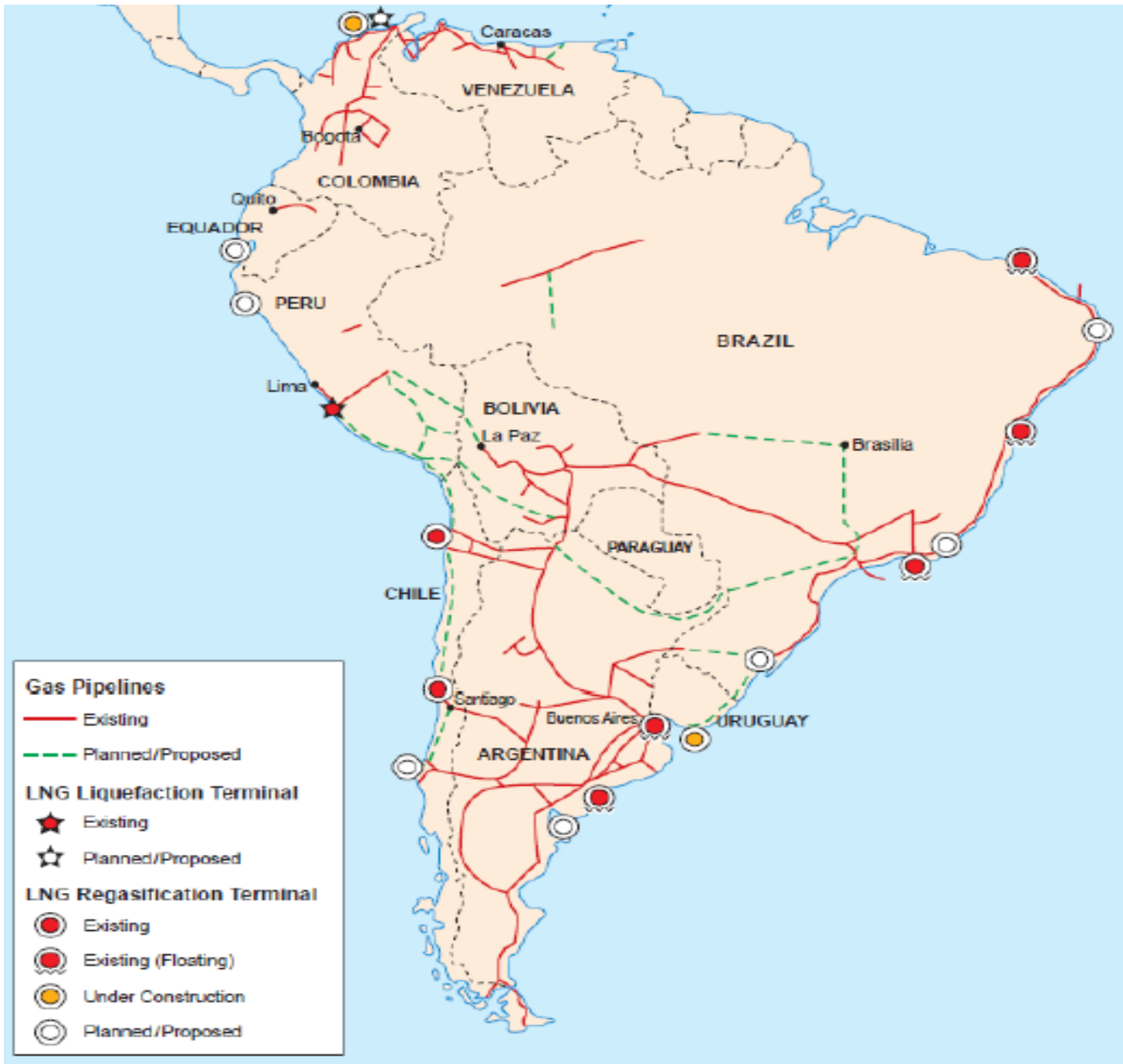

Fuente: Oxford Institute for Energy (2016).

\section{Infraestructura de interconexiones de electricidad}

Así como la infraestructura gasífera, la infraestructura integrada del sistema eléctrico es fundamental para la integración física. La figura 3, muestra las líneas de transmisión de electricidad existentes en América del Sur al año 2020. Se observa que en la región del Gran Chaco existen 5 líneas de interconexión, de las cuales solamente dos están operando (Referencias 29 y 30 en Tabla 2): una corresponde a la Central hidroeléctrica Yacyretá y la otra está en la frontera Argentina-Paraguay; la línea Juana Azurduy (Referencia 16), ubicada en la frontera Bolivia-Argentina, se encuentra en fase de construcción; y existen además dos líneas que se encuentran en fase de estudio (Referencias 14 y 15), que conectan Bolivia-Brasil y Bolivia-Paraguay respectivamente. 
Figura 3: Mapa Líneas de Transmisión CIER, 2020.

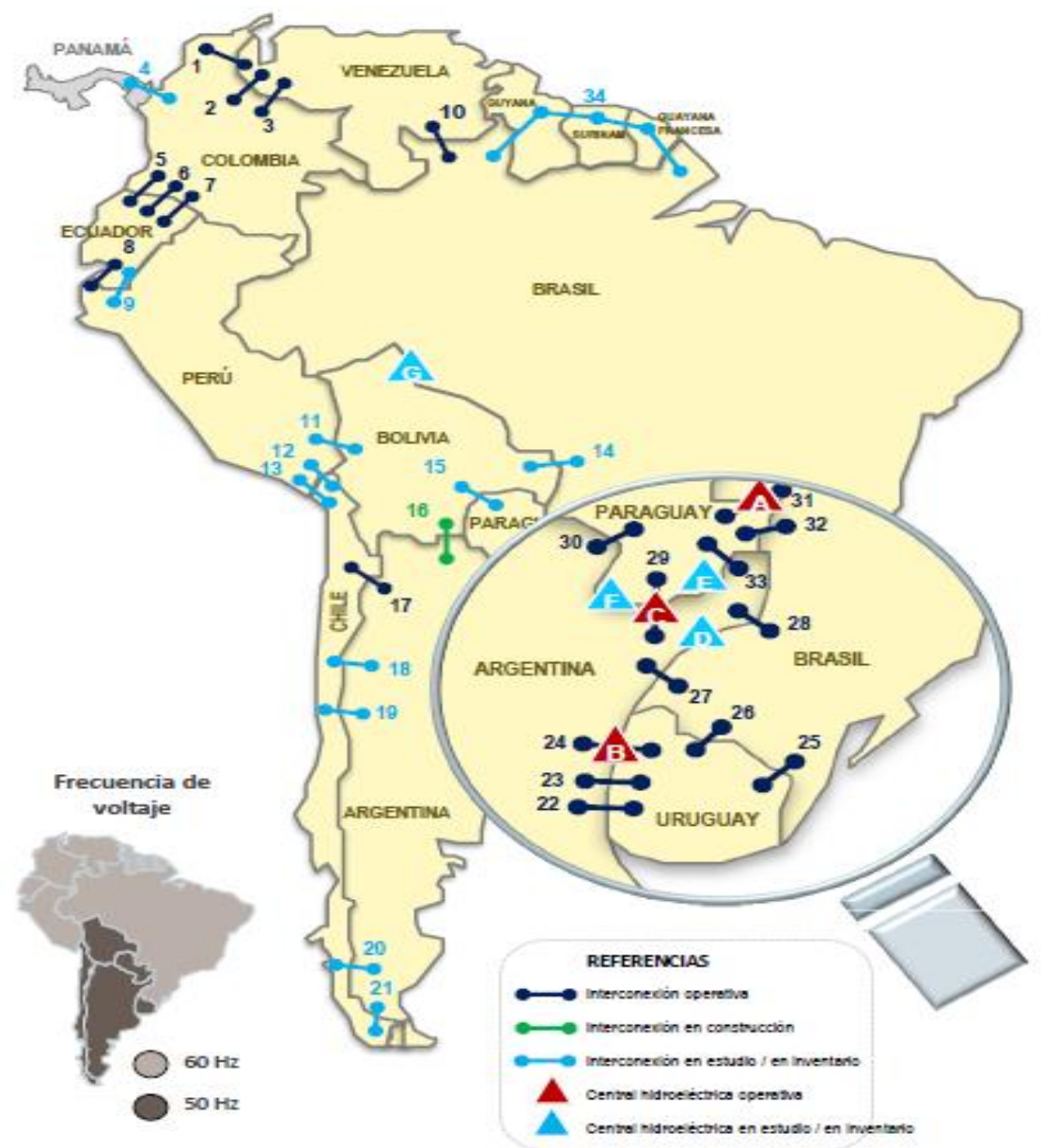

Fuente: Comisión de Integración Regional (2020)

Tabla 2: Interconexiones eléctricas área Gran Chaco y zonas aledañas

\begin{tabular}{|c|c|c|c|c|c|}
\hline Ref. & Países & Ubicación & Tensiones & Potencia & Observaciones \\
\hline 14 & Bo-Br & $\begin{array}{l}\text { Interconexión Bolivia - } \\
\text { Brasil }\end{array}$ & 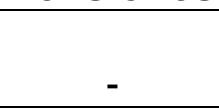 & 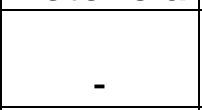 & $\begin{array}{l}\text { En estudio } \\
\text { (ENDE/ELETROBRAS) }\end{array}$ \\
\hline 15 & Bo-Py & $\begin{array}{l}\text { Interconexión Bolivia - } \\
\text { Paraguay }\end{array}$ & - & - & En estudio (ENDE/ANDE) \\
\hline 16 & Ar-Bo & $\begin{array}{l}\text { Yaguacua (Bo) - Tartagal } \\
\text { (Ar) (Juana Azurduy) }\end{array}$ & $132 \mathrm{kV}$ & $120 \mathrm{MW}$ & $\begin{array}{l}\text { En construcción }(50 \mathrm{~Hz}) \\
46 \mathrm{~km} \text { (Bolivia)/ } 64 \mathrm{~km} \\
\text { (Argentina) }\end{array}$ \\
\hline 29 & Ar-Py & $\begin{array}{l}\text { Salidas de Central } \\
\text { Yacyretá }\end{array}$ & & $\begin{array}{l}3.200 \\
\mathrm{MW}\end{array}$ & Operativa $(50 \mathrm{~Hz})$ \\
\hline 30 & Ar-Py & $\begin{array}{l}\text { Clorinda (Ar) - } \\
\text { Guarambaré (Py) }\end{array}$ & $\begin{array}{c}132 / 220 \\
\mathrm{kV}\end{array}$ & $\begin{array}{l}80 / 90 \\
\mathrm{MW}\end{array}$ & $\begin{array}{l}\text { Operativa de oportunidad } \\
(50 \mathrm{~Hz})\end{array}$ \\
\hline
\end{tabular}

Fuente: Comisión de Integración Regional (2020) 
Tabla 3: Interconexiones eléctricas menores área Gran Chaco y áreas aledañas

\begin{tabular}{|l|l|l|l|}
\hline Países & Ubicación & Tensión & Observaciones \\
\hline Ar-Bo & La Quiaca (Ar) - Villazón (Bo) & $13,2 \mathrm{kV}$ & Existente \\
\hline Ar-Bo & Pocitos (Ar) - Yacuiba (Bo) & $33 \mathrm{kV}$ & Existente \\
\hline Ar-Py & Posadas (Ar) - Encarnación (Py) & $33 \mathrm{kV}$ & No operativa \\
\hline Ar-Uy & Concordia (Ar) - Salto (Uy) & $30 \mathrm{kV}$ & No operativa \\
\hline $\mathrm{Bo}-\mathrm{Br}$ & Puerto Suárez (Bo) - Corumbá (Br) & $13,8 \mathrm{kV}$ & Existente \\
\hline $\mathrm{Bo}-\mathrm{Br}$ & San Matías (Bo) - Corixa (Br) & $35 \mathrm{kV}$ & Operativa \\
\hline $\mathrm{Br}-\mathrm{Py}$ & $\begin{array}{l}\text { Ponta Pora (Br) - Pedro Caballero } \\
(\mathrm{Py})\end{array}$ & $22 \mathrm{kV}$ & Existente, 6 MW \\
\hline
\end{tabular}

Fuente: Comisión de Integración Regional (2020)

Tabla 4: Centrales binacionales en áreas cercanas al Gran Chaco

\begin{tabular}{|l|l|l|l|l|l|}
\hline Ref. & Países & Denominación & Río & Capacidad instalada & Observaciones \\
\hline $\mathrm{C}$ & $\mathrm{Ar}-\mathrm{Py}$ & Yacyretá & Paraná & $3.200 \mathrm{MW}$ & En operación \\
\hline $\mathrm{F}$ & $\mathrm{Ar}-\mathrm{Py}$ & Itatí - Itá Corá & Paraná & $1.600 \mathrm{MW}$ & En inventario \\
\hline
\end{tabular}

Fuente: Comisión de Integración Regional (2020)

La Tabla 3 presenta algunas interconexiones eléctricas menores en la zona del Gran Chaco y áreas aledañas que podrían transportar la energía a la región. La Tabla 4 muestra que también existen centrales binacionales importantes que están en el área, principalmente la central hidroeléctrica Yacyretá. La central Itaipú se encuentra fuera del área chaqueña, sin embargo, una parte significativa de la energía que se consume en Paraguay proviene de esa central.

\section{Perspectivas para la integración de la infraestructura energética}

Según OIES (2016), satisfacer las necesidades tanto de generación adicional como de flexibilidad adicional, será uno de los mayores retos para impulsar la integración gasífera. Se espera que la demanda de energía continúe amentando conforme crece la población y los niveles de actividad económica en la región. Esto se puede percibir en las interconexiones que se encuentran en fase de estudio, que se presentan como promisorias de un mayor nivel de actividad en la región del sur de Bolivia, sur-oeste de Brasil y norte de Paraguay.

Se espera que el gas natural cumpla un papel preponderante en los siguientes años, especialmente en la generación de electricidad. De acuerdo a Morales Udaeta (et al., 2006), la importancia creciente del mismo en la matriz energética de las naciones del Cono Sur, debido al tamaño de las reservas y a la demanda creciente será cada vez más significativa para garantizar el abastecimiento del gas natural en la región. El GNL también podría tener un 
aumento en la demanda dado que representa otra alternativa, sin embargo, no requiere cooperación trasfronteriza.

Aunque el gas natural es un insumo no renovable, no se deben desconsiderar las nuevas normativas que pueden surgir a partir de los requerimientos de diversos organismos internacionales que promueven el combate al calentamiento global y restringen actividades que producen gases de efecto invernadero o promueven uso de energías renovables y más limpias ${ }^{89}$. Las fuentes renovables tienen un gran papel en la actual matriz energética de América del Sur, sin embargo, el Gran Chaco no cuenta con disponibilidad de algunas de estas fuentes. No tiene potencial para producir biomasa, tampoco cuenta con grandes niveles de radiación solar ni vientos con velocidad adecuada para generar energía eólica ${ }^{10}$.

Uno de los principales desafíos de los gobiernos para fomentar el aprovechamiento compartido de los recursos y la infraestructura es a través del fortalecimiento de las instituciones de Integración Regional. De acuerdo con Padula (2012), a pesar de la existencia de instituciones como IIRSA, que procuraron impulsar algunos proyectos de integración energética, los países de la región suramericana no han aprovechado sus complementariedades energéticas, potenciales y actuales, en pro de una política de desarrollo y seguridad energética regional. Actualmente, otras instituciones o proyectos pueden llegar a tener más influencia en las decisiones relacionadas a la creación o ampliación de infraestructura energética y en la gobernanza de los recursos energéticos compartidos. Por ejemplo, la Zona de Integración del Centro Oeste de Sudamérica (ZICOSUR), se constituye como una instancia política de integración de los gobiernos sub-nacionales, que busca incrementar el intercambio comercial y económico con Asia y otros mercados del Pacífico, aprovechando mejor la potencialidad productiva del área sudamericana (Zicosur, 2018). De esa manera podría motivar el interés por la integración de la infraestructura energética regional. El proyecto del corredor bioceánico también podría cumplir un papel similar.

\footnotetext{
${ }^{8}$ Por ejemplo, desde1996, Argentina, Bolivia y Paraguay han firmado varios acuerdos acerca del Gran Chaco, incluyendo una Declaración entre los Puntos Focales Nacionales, la Convención de Desertificación (UNCCD) y el mecanismo global, estableciendo así el marco para la cooperación regional para el desarrollo sostenible del Gran Chaco. La declaración se enfocó en la mejora de las condiciones socioeconómicas de las poblaciones locales y el establecimiento de acciones concretas para mitigar la degradación de los ecosistemas en el Chaco (http://www.oas.org/dsd/WaterResources/projects/Chaco_esp.asp)

${ }^{9}$ Algunos ejemplos son la creación de un Consejo Plurinacional para Vivir Bien en Armonía y Equilibrio con la Madre Tierra y los Mecanismos de Mitigación para Vivir Bien en Bolivia (Investigación para el Desarrollo, 2017).

${ }^{10}$ http://servicios.infoleg.gob.ar/infolegInternet/anexos/140000-144999/141453/norma.htm
} 


\section{Consideraciones finales}

Este trabajo tuvo por objetivo analizar la situación actual y las perspectivas de la integración de la infraestructura energética en la región del Chaco Americano, destacando su relevancia para la integración regional suramericana. Algunas de las herramientas teórico-conceptuales de la geopolítica energética empleadas para el análisis, permitieron visualizar con mayor claridad la centralidad de la energía en el ámbito de las relaciones internacionales, siendo ésta un recurso de poder, que es naturalmente conflictivo, pero que también puede servir de base para una mayor cooperación e integración regional y la búsqueda de autosuficiencia y seguridad energética.

La riqueza en recursos gasíferos del Gran Chaco y la centralidad de Bolivia en la geopolítica energética dentro de la región denominada Heartland suramericano, hacen que ésta sea una zona clave para impulsar el proceso de integración regional a través de la estrategia de un mayor control de los recursos energéticos. Además, porque allí confluyen áreas geográficas de países que comparten interconexiones eléctricas, gasíferas y otras infraestructuras. Para un análisis más completo, es necesario estudiar la evolución de los flujos comerciales de electricidad y gas, así como de las necesidades energéticas que se prevén en la zona, a partir de los mayores flujos comerciales que pueden circular por los nuevos corredores. No obstante, con la información disponible sobre la situación de la infraestructura energética, se prevé que habrá un mayor flujo comercial de electricidad y de gas, debido a los gasoductos e interconexiones que se encuentran en fase de construcción o de estudio.

Aunque la región aún está lejos de contar una política energética regional con base en un proyecto planificado y coordinado, el fortalecimiento institucional y los mecanismos de cooperación eficientes son claves. Éstos pueden evitar que algunos países dependientes de la importación de gas (o de electricidad) empleen estrategias de seguridad en el abastecimiento externo que acaben afectando a los países exportadores. Por otro lado, pueden promover la creación o ampliación de infraestructura con marcos legales regionales que faciliten el uso y la gestión compartida de recursos.

\section{Bibliografía}

Aguilera Cazalbón, Astrid (2020). Integración Energética Regional: Transición Energética en la Coyuntura Actual. La Espalda, Revista do Observatório da Integração Econômica da América do Sul - Unila. Pág. 20 y 21, Mayo de 2020, Volúmen 22. Foz do Iguaçu - PR 
Gomes Carmona, Ronaldo (2020). Brasil, Potência Energética. Notas sobre Geopolitica e Energia. Em: Geopolítica e Energia. Synergia Editora, São Paulo.

Conant, Melvin A.; Gold, Fern R. (1981). A Geopolítica Energética. Biblioteca do Exército Editora, Rio de Jeneiro, Brasil.

Centro Brasileiro de Infraestrutura (2020). O que é o Gasbol?. CBIE, 4 Setiembre de 2020. Disponible en: https://cbie.com.br/artigos/o-que-e-o-gasbol/

Empresa de Pesquisa Energética (2017). Panorama da Indústria de Gás Natural na Bolívia. EPE, Rio de Janeiro, 22 de junho de 2017.

Fuser, Igor (2013). Energia e Relações Internacionais. São Paulo: Editora Saraiva.

Hémery, Daniel; Debeir, Jean-Claude \& Deléage, Jean-Paul (1993). Uma História da Energia. Ed. UnB: Brasília, DF.

Investigación para el Desarrollo (2017). Evaluación de Vulnerabilidad e Impacto del Cambio Climático en el Gran Chaco Americano. Serie Clima y Recursos Naturales, Número 1, año 2017.

Kerr de Oliveira, Lucas.; García Leite, Tatiana. (2010). O conceito de Heartland na geopolítica clássica: funcionalidade e limites para a análise da Região central da América do Sul. V Encontro Nacional da ANPPAS, Florianópolis.

Kerr de Oliveira, Lucas (2012). Energia como recurso de poder na política internacional: geopolítica, estratégia e o papel do Centro de Decisão Energética. Tese de Doutorado. PPGCP. Porto Alegre: UFRGS, 2012.

Mello, Leonel Itaussu de Almeida. Quem tem medo da Geopolítica? São Paulo: Edusp, 1999.

Mackinder, Halford J. (2010) El pivote geográfico de la historia. CLÁSICOS GEOPOLÍTICOS. Traducción de Marina Díaz Sanz. Geopolítica(s) 2010, vol. 1, núm. 2, 301-319.

Morales Udaeta, Miguel; Oliva Rocha, Cidar R.; Orellana Lafuente, Renan; Rojas Zurita, Ramiro (2006). La integración gasífera energética vía CAMISEA por el lado del Pacifico. Congresso Brasileiro de Planejamento Energético. Políticas públicas para a Energia: Desafios para o próximo quadriênio, Brasília.

Oxford Institute for Energy Studies (2016). South American Gas Market sand the Role of LNG. OIES paper: NG 114. October 2016, Oxford.

Padula, Raphael (2010). Integração Regional de Infraestrutura e comercio na América do Sul nos anos 2000: Uma análise político-Estratégica. Tese de Doutorado, COPPE-UFRJ. Rio de Janeiro, 2010.

Padula, Raphael (2012). Una concepción de la Integración Regional, el papel de la Infraestructura y de la ocupación de espacios. Pág. 207-288. En: COSTA, D.; DA PAZ, A.; NUNES, R.; PADULA, R.; WEXELL SEVERO, L. 2012. América del 


\section{Universidade Federal Fluminense Instituto de Estudos Estratégicos}

Sur. Integración e infraestructura. Capax Dei Editora. Brasil, Rio de Janeiro, 2012.

Paz, A; Nunes, R. (2011). Buscando as complementariedades. Diagnósticos das Interconexões Energéticas Sul-Americanas. P (99-142). In: COSTA, D.; DA PAZ, A.; NUNES, R.; PADULA, R.; WEXELL SEVERO, L. 2011. América do Sul. Integração e infraestrutura. Capax Dei Editora. Brasil, Rio de Janeiro, 2011

Sauer, Ildo (2015). Proposta de integração produtiva em energia: recursos minerais para o desenvolvimento na América Latina. Pag. 173-197. En: Os Desafios da América Latina no Século XXI. Enrique Iglesias (coordenação); organização, Gerson Damiani, Adolfo Garcé, Fabíola Wüst Zibetti. São Paulo, Editora da Universidade de São Paulo, 2015

Transnational Institute (2019). Towards a Corporate or a Peoples' Energy Transition? in: Energy Transition. contributions for collective reflection, Transnational Institute (TNI) and Taller Ecologista, Diciembre de 2019.

Wxell Severo, Luciano (2012). A importância geopolítica da Bolívia e a integração da América do Sul. (pp. 137-139). Em: América Andina. Integração regional, segurança e outros olhares. Organizadores: Renata Peixoto de Oliveira, Renata; Garcia Nogueira, Silvia; Reis Melo, Felipe. Editorial Eduepb, Campina Grande, São Paulo.

Zicosur (2018). Zona de Integración del Centro Oeste de Suramérica. Áreas de Gestión Compartidas. Integración política regional en los territorios. Disponible en:http://zicosur.co/wpcontent/uploads/2018/05/ AREASDE GESTION

COMPARTIDA.pdf 